\title{
IT-Sicherheit und Datenschutz: Nutzergerecht
}

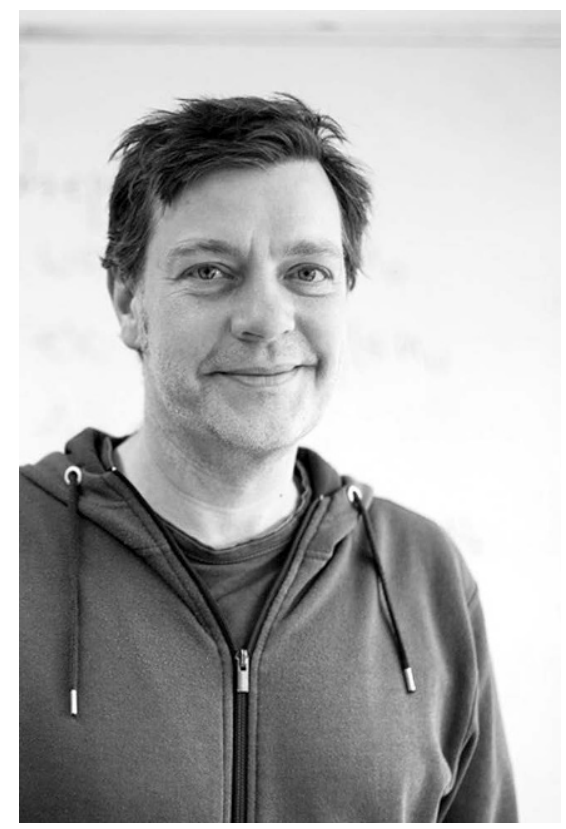

In der realen Welt haben wir über Jahrtausende gelernt, Gefahren zu erkennen und richtig darauf zu reagieren. Häufig handeln wir hier intuitiv, d.h. wir müssen über unser Handeln nicht lange nachdenken. Ganz anders ist die Situation, wenn wir uns in der digitalen Welt bewegen. So etwas wie knacken im Wald oder Mimik von Gesprächspartnern gibt es hier nicht. Insofern ist der viel belächelte Satz "Das Internet ist für uns alle Neuland", den Frau Merkel 2013 im Zuge der Snowden-Affäre sagte, auch heute noch richtig. Es wird sicherlich noch einige Zeit dauern, bis wir uns souverän in der digitalen Welt bewegen können. Wichtig ist, dass uns die von uns genutzten Produkte dabei unterstützen.

Daher freue ich mich, dass mir die Gelegenheit gegeben wurde, in diesem Heft genau diese Themen aufzugreifen. Mittlerweile hat sich hier der Begriff Usable Security and Privacy etabliert. Dies zeigt auch, dass das Thema zunehmend an Bedeutung gewinnt. In dieser Ausgabe wird das Thema aus unterschiedlichen Perspektiven beleuchtet. So schlägt z.B. Christian Aretz in seinem Aufsatz vor, zentrale Vertrauensplattformen zu nutzen, um wieder Herr über seine Daten zu werden. Dieses Konzept wird auch in dem Artikel von Niclas Nüske, Christian Olenberger, Daniel Rau und Fabian Schmied als Erweiterung ihrer Idee eines Privacy Bots diskutiert. Privacy Bots sollen Datenschutzerklärungen automatisch auswerten und so Nutzerinnen und Nutzer beispielsweise Warnhinweise geben können. Andreas Heinemann und Tobias Straub schlagen vor, die Erkenntnisse aus dem Forschungsgebiet Usable Privacy and Security für benutzerfreundliche Datenschutzlösungen zu nutzen. Sie diskutieren in ihrem Beitrag mehrere Konzepte aus diesem Bereich, um den mit den Betroffenenrechten verbundenen Herausforderungen begegnen zu können.

Eine weitere wichtige Herausforderung hinsichtlich Benutzbarkeit ist, dass Nutzerinnen und Nutzer Sicherheit und Datenschutz ohne zusätzliche Hardwarekomponenten umsetzen können. Moderne Smartphones bringen schon zahlreiche Sicherheitsfunktionen mit sich, womit sich z.B. digitale Identitäten sicher anwenden lassen, wie der Beitrag von Tim Ohlendorf, Wolfgang Studier und Marian Margraf zeigt. Datenschutz und Sicherheit fängt aber schon beim Betreiber einer Lösung an. Wie sich diese Themen auch in kleinen Institutionen aus Wirtschaft und Verwaltung benutzerfreundlich und sicher umsetzen lassen, zeigt der Beitrag von Stefan Pfeiffer und Martin Seiffert. Hier sind die Zielgruppen nicht Nutzerinnen und Nutzer, sondern IT-Sicherheits- und Datenschutzbeauftragte.

Die Benutzbarkeit von Sicherheit und Datenschutz wird seit Mitte der 90er Jahre vermehrt in der Wissenschaft diskutiert. Es zeigt sich, dass einige der in der Wissenschaft entwickelten Konzepte nun auch in Produkte aufgenommen werden. Es liegt aber noch ein weiter Weg vor uns.

Zusätzlich zum Heftschwerpunkt werden von Ruth-Stock Homburg und Christian Holthaus Aspekte des Privacymanagement in Unternehmen behandelt.

Wir wünschen Ihnen viel Freude und interessante Erkenntnisse beim Studium der Beiträge dieser DuD-Ausgabe. Auch über ein Feedback würden wir uns sehr freuen.

\section{Marian Margraf}

